\title{
Iridium-Catalyzed Reductive Allylation of Esters
}

\author{
Lan-Gui Xie, Jack Rogers, ${ }^{\ddagger}$ Ioannis Anastasiou, ${ }^{\ddagger}$ Jamie A. Leitch, ${ }^{\ddagger}$ Darren J. Dixon. ${ }^{\ddagger}$ \\ $\ddagger$ Department of Chemistry, University of Oxford, Chemistry Research Laboratory, 12 Mansfield Road, Oxford, OX1 3TA, \\ $\mathrm{UK}$
}

KEYWORDS Allylation, Ether, Ester, Lactone, Silane, Reductive

\begin{abstract}
The catalytic reductive transformation of carboxylic esters into $\alpha$-branched ethers is described. The procedure pivots on the chemoselective iridium-catalyzed hydrosilylation of ester and lactone functionality to afford a silyl acetal intermediate. Upon treatment with Lewis acid these hemi-labile intermediates ionize to form reactive oxocarbenium ions, which can be intercepted by allyl tributyltin nucleophiles, resulting in the formation of valuable $\alpha$-branched alkyl-alkyl ether derivatives. This reductive allylation procedure was amenable to a range of carboxylic ester starting materials and good chemoselectivity for ethyl over $t$-butyl esters was demonstrated. Furthermore, downstream synthetic manipulation of $\alpha$-amino acid derived products led to efficient formation of pyrrolidine, piperidine, and azepane frameworks.
\end{abstract}

The $\alpha$-branched alkyl-alkyl ether motif is prevalent in many biologically active structures and industrially relevant materials. Accordingly, the development of new, effective, and practical protocols to synthesize such fragments is important. ${ }^{1}$ Although traditional and well-established synthetic methods to substituted ethers - including Williamson ether synthesis, hydroalkoxylation of alkenes, and $\mathrm{C}-\mathrm{O}$ bond cross coupling to name a few ${ }^{2}-$ are still routinely used, in recent years the development of complementary, chemoselective, mild and efficient ways for their preparation has attracted substantial interest, including pioneering photocatalytic and $\mathrm{C}-\mathrm{H}$ functionalization techniques. ${ }^{3}$ In particular, direct $\alpha-\mathrm{C}-\mathrm{H}$ oxidative coupling (cross-dehydrogenative coupling) of ether derivatives and nucleophiles (Scheme 1A) has become a powerful complementary approach to classical routes. ${ }^{4}$ From a synthetic standpoint, we recognized that the ability to access similar architectures from corresponding feedstock, commercially available, or readily synthesized carboxylic esters - using a catalytic reductive protocol - could provide a powerful alternative towards these desirable complex ether products.

Due to its relatively low electrophilicity, direct nucleophilic addition to ester functionality generally requires reactive organometallic nucleophiles (such as organomagnesium and organolithium reagents) and typically results in the formation of tertiary alcohols. Furthermore, long-standing interest concerning ester transformations has focused on the use of aluminum acetal intermediates, generated by partial reduction of esters with DIBAL. The activity of this intermediate allows the coupling with various carbon-centred nucleophiles in the presence of Lewis acids, either directly or via acetylated acetal intermediates, affording secondary alcohols or cyclic ethers as products. ${ }^{5,6}$ Conversely, the transition metal catalysed hydrosilylation of an ester to a silyl acetal under mild conditions has also been achieved. ${ }^{7}$ However, the use of silyl acetal intermediate for carbon-carbon bond formation has been largely overlooked, ${ }^{7 \mathrm{~m}}$ especially towards the synthesis of alpha branched ether fragments. ${ }^{8}$
We envisaged that a selective reductive transition metalcatalyzed hydrosilylation of an ester would create the silyl acetal, which under the activation of an appropriate Lewis acid would afford the corresponding oxocarbenium ion (Scheme 1B). Interception of this reactive intermediate with a suitable carbon-centered nucleophile would then afford the $\alpha$-branched ether in a two-step, one-pot procedure. Such an efficient, catalytic, chemoselective, and general approach to the reductive transformation of ubiquitous esters to high value $\alpha$-branched ethers could be of importance to synthetic development programmes, and herein we wish to report our findings.

\section{Scheme 1. Catalytic construction of $\alpha$-alkyl ethers.}

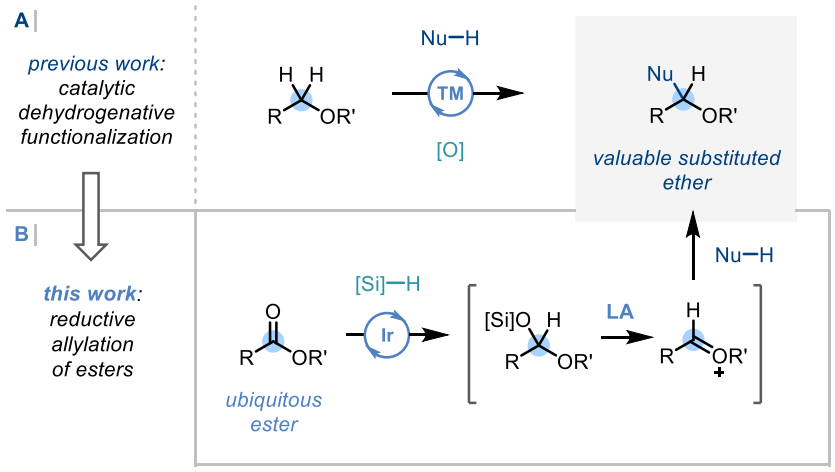

We selected the reductive allylation of ethyl 4bromobenzoate (1a) using allyl tributyltin (2a) as our model reaction. $\left[\operatorname{Ir}(\mathrm{coe})_{2} \mathrm{Cl}\right]_{2}$ was employed as catalyst and diethylsilane as the reducing agent, ${ }^{7}$ and initial studies using toluene as solvent delivered smooth hydrosilylation of the ester. Subsequent functionalization with $\mathbf{2 a}$ (2 equiv) using $\mathrm{BF}_{3} \cdot \mathrm{Et}_{2} \mathrm{O}$ (1.1 equiv) as a Lewis acid additive afforded promising yields of the desired $\alpha$-allylated ether product 3a (Table 1 , entry 1 ). 
Table 1. Optimization of the iridium-catalyzed reductive allylation of esters

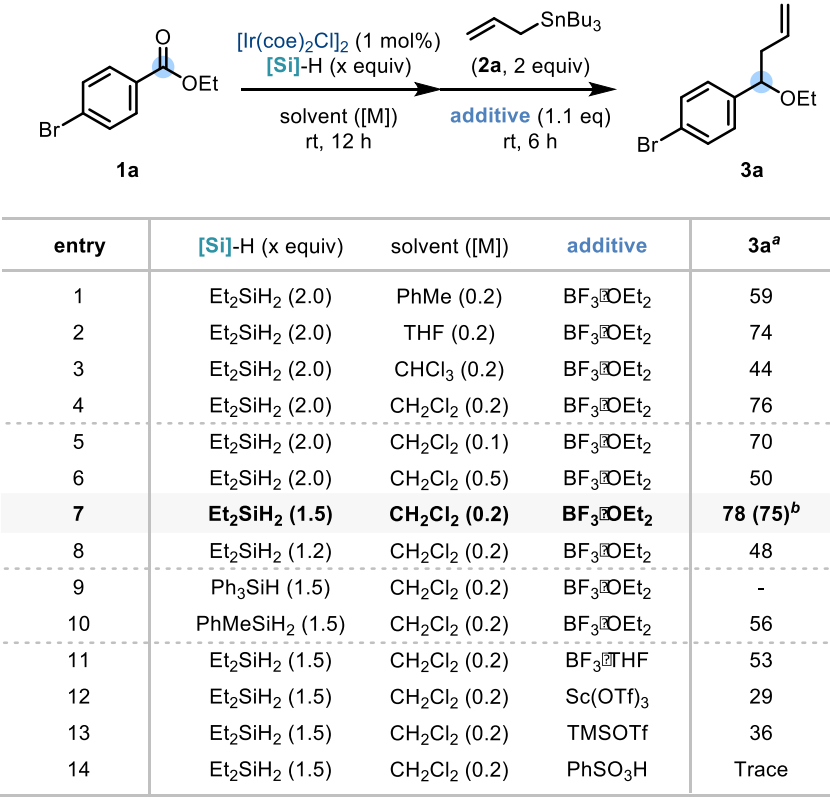

General conditions. Step (i): ethyl 4-bromobenzoate (1a, $0.3 \mathrm{mmol}),\left[\operatorname{lr}(\mathrm{coe})_{2} \mathrm{Cl}\right]_{2}(1 \mathrm{~mol} \%)$, solvent $[M]$, under an $\mathrm{N}_{2}$ atmopshere at room temperature for $12 \mathrm{~h}$. Step (ii): $2 \mathrm{a}(0.6 \mathrm{mmol})$, additive (1.1 eq) under an $\mathrm{N}_{2}$ atmosphere at room temperature for $6 \mathrm{~h}$. ${ }^{a}$ Formation of 3a was calculated via ${ }^{1} \mathrm{H}$ NMR analysis of the crude reaction mixture against 4-dimethylaminopyridine as an internal standard. ${ }^{b}$ Isolated yield after silica gel column chromatography.

Further experiments identified dichloromethane as the solvent of choice (entries 2-4). Pleasingly, reducing the amount of $\mathrm{Et}_{2} \mathrm{SiH}_{2}$ (from $2-1.5$ equiv) was shown to be inconsequential to reaction proficiency, delivering $\alpha$-allylated product in $75 \%$ yield (entry 7). However, further reduction in equivalents of $\mathrm{Et}_{2} \mathrm{SiH}_{2}$ to 1.2 led to decreased efficiency (entry 8). Interestingly other silane reagents - exemplified with $\mathrm{Ph}_{3} \mathrm{SiH}$ and $\mathrm{PhMeSiH}_{2}$ - showed substantially poorer performance in this transformation (entries 9-10). Furthermore, other Lewis acids were shown to be detrimental to reaction efficiency (including $\mathrm{BF}_{3} \cdot \mathrm{THF}$, entries 11-13). Brønsted acids such as $\mathrm{PhSO}_{3} \mathrm{H}$ (entry 14) were ineffective, and notably in this case, the amount of competitive over-reduction products was significantly increased. ${ }^{9}$

With optimal conditions established, we then explored the scope of this reductive allylation methodology by submitting a range of esters to the reaction conditions. ${ }^{10}$ Variation of the alkoxy part of the ester including ethyl (3a, Scheme 2), isopropyl (3b), butyl (3h), and notably methyl (3i, previously predominately giving competitive hydrolysis products $)^{8}$ esters were demonstrated to form the corresponding allylated ether products in good yields. Esters bearing benzyl ether (3d), aryl ether (3e), secondary amine (3f) and carbamate (3g) functionalities were also amenable to the reductive allylation protocol, delivering the corresponding $\alpha$-allyl substituted ethers in excellent efficiency - with a glycine derived substrate shown to perform effectively on gram-scale (3f). Pleasingly, free hydroxyl containing esters also proved applicable to this protocol (3g-j). We then looked to cyclic lactones as viable substrates, where $\omega$-pentadecalactone $(\mathbf{3 k}),( \pm) \delta$-undecalactone $(\mathbf{3 l})$, and D-glucono lactone $(\mathbf{3 m})$ proceeded in moderate to good yields and excellent diastereoselectivities (where applicable). ${ }^{11}$

Furthermore, subsequent studies demonstrated that variation of the allyl tributyltin reagent was also tolerated, enabling the synthesis of homologous allyl substituted ethers. Thus, the addition of methallyltributyltin to the generated silyl hemiacetal intermediate allowed the proficient synthesis of $\mathbf{3 f a}$. Sterically demanding organostannanes - exemplified by tributyl(3methyl-2-butenyl)tin - were also applicable, furnishing the corresponding $\alpha$-branched ether 3 fc in $69 \%$ yield. Furthermore, the compatibility of tributyl(cyclohex-1-en-1ylmethyl)tin led to the installation of cyclohexenyl functionality $(\mathbf{3 f c})$. Encouraged by the success of the scope with respect to both reaction partners, we then submitted unsymmetrical malonic acid diester substrate (3o) to this reductive allylation protocol. Selective reductive allylation of the ethyl ester took place with tert-butyl ester functionality remaining largely intact, affording ether product in 57\% yield, demonstrating the steric preference in the hydrosilylation step.

We recognized that this chemistry and the ethers they produce could provide new avenues into $\mathrm{sp}^{3}$-rich heterocycle synthesis. The products of this reductive allylation methodology could readily enable the construction of decorated 5-, 6-, and 7-membered nitrogen-containing heterocycles from simple amino acid precursors (Scheme 3). Identifying $\mathbf{3 f}$ as a synthetic lynchpin, first a two-step $N$-allylation, ring closing metathesis sequence gave the unsaturated azepane (4a, see supporting information for full reaction details $)^{12}$. Next, a tandem hydroboration, ${ }^{13}$ palladium-catalyzed amination protocol gave the substituted piperidine in excellent yields for a two-step process (4b). Finally we envisaged that $\mathbf{3 f}$ could undergo palladiumcatalyzed aminoarylation to give the corresponding substituted pyrrolidine. ${ }^{14}$ Pleasingly, using 2-naphthylbromide as a coupling partner, this was indeed achieved in good yields to create the 5-membered heterocycle (4c).

In conclusion, an iridium-catalyzed reductive allylation of esters for the synthesis of $\alpha$-branched ethers, by coupling of in situ generated silyl hemiacetal intermediate and allyltributyl tin derivatives, has been developed. This reaction benefits from the strategic use of stable and readily available esters as abundant and under-exploited ether precursors. The broad scope of substrates that can be tolerated under the mild reductive conditions demonstrates the practicality of this new carbon-carbon bond formation reaction. In combination with the illustrated synthetic utility by derivation of the ether products, this $\alpha$-allyl substituted ether synthesis protocol will likely find use in selective syntheses of simple and complex ethers alike. 
Scheme 2. Substrate scope for the reductive allylation of esters

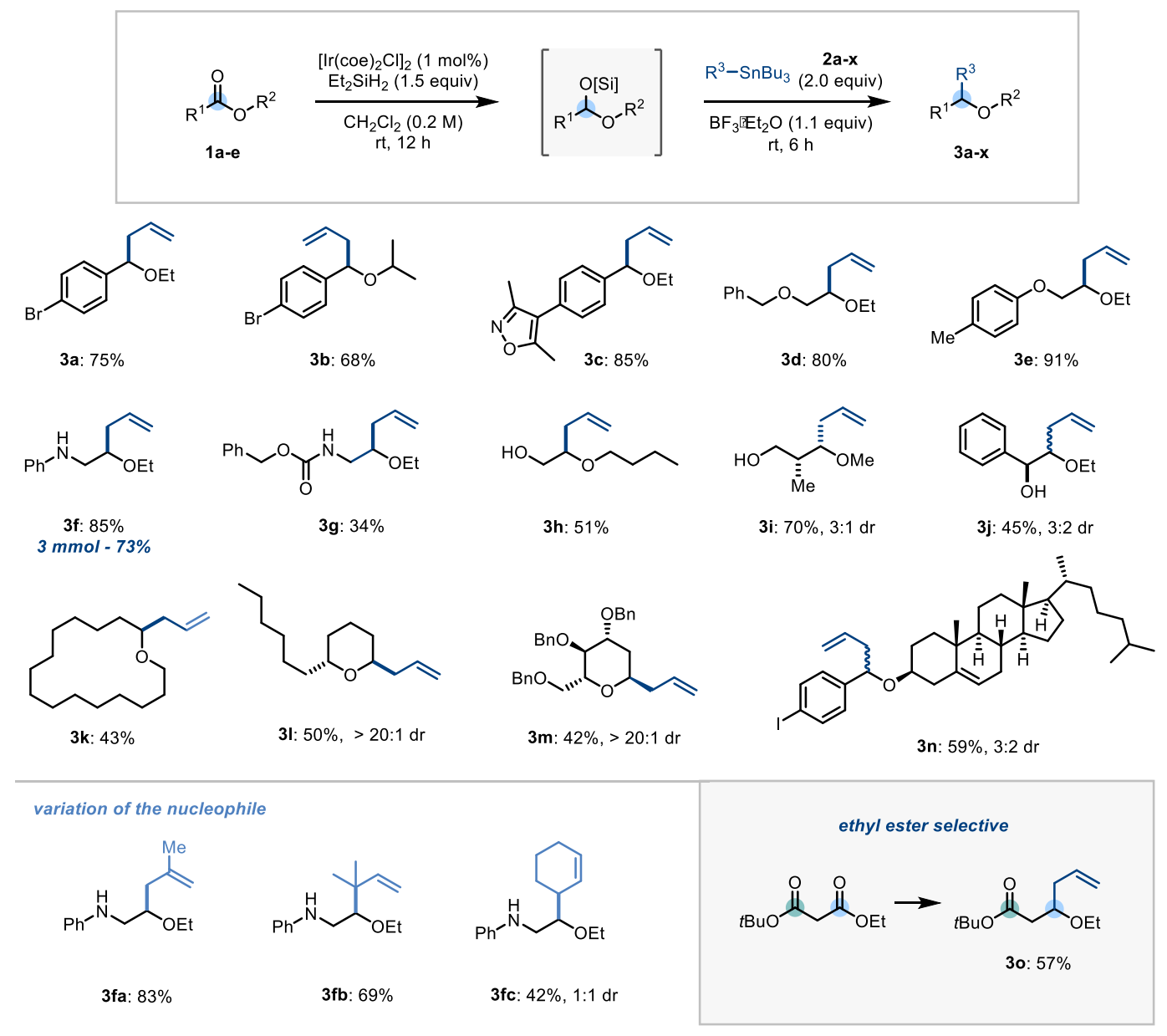

Scheme 3. Applications in heterocycle synthesis

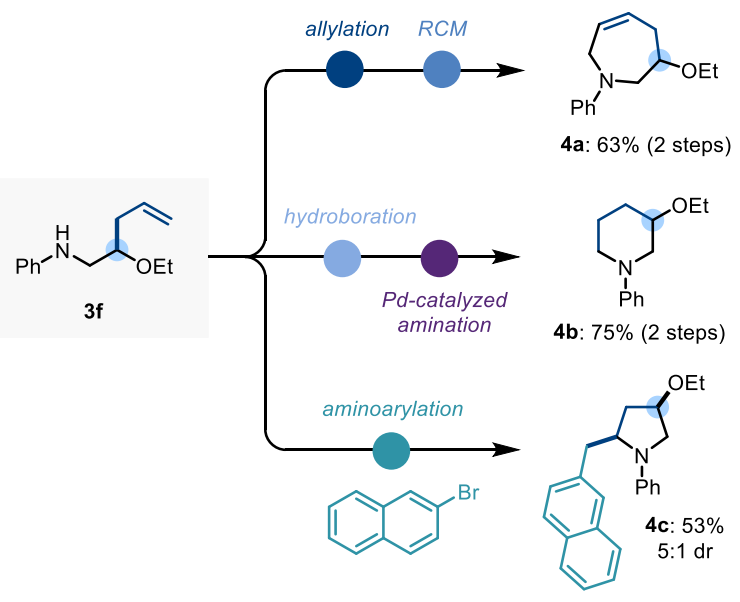

- allylbromide (4 eq), $\mathrm{Na}_{2} \mathrm{CO}_{3}(0.6 \mathrm{eq}), \mathrm{EtOH} / \mathrm{H}_{2} \mathrm{O}$ - Grubbs I (5 mol\%), $\mathrm{CH}_{2} \mathrm{Cl}_{2}$

9-BBN (3 eq), THF

- $\mathrm{Pd}\left(\mathrm{PPh}_{3}\right)_{4}(10 \mathrm{~mol} \%), 2-\mathrm{BrPy}(1.2 \mathrm{eq}), \mathrm{K}_{2} \mathrm{CO}_{3}(4 \mathrm{eq}), \mathrm{H}_{2} \mathrm{O}, \mathrm{DMF}$

- $\mathrm{Pd}_{2}(\mathrm{dba})_{3}(10 \mathrm{~mol} \%)$, Xantphos (15 mol\%), NaOtBu (2 eq), PhMe

\section{ASSOCIATED CONTENT}

The Supporting Information is available free of charge on the ACS Publications website. Synthetic procedures and full characterization data of compounds (PDF).

\section{AUTHOR INFORMATION}

\section{Corresponding Author}

*darren.dixon@chem.ox.ac.uk

\section{ACKNOWLEDGMENT}

LGX thanks Marie Curie Actions for a fellowship (H2020MSCA-IF-2015, 707559). JAL would like to thank the Leverhulme Trust (RPG-2017-069) for a research fellowship.

\section{REFERENCES}

(1) (a) See corresponding chapters in Ullmann's Encyclopedia of Industrial Chemistry, Wiley-VCH, Weinheim, 2011, 7th edn.; (b) Blunt, J. W.; Copp, B. R.; Munro, M. H. G.; Northcote, P. T.; Prinsep, M. R. Marine natural products. Nat. Prod. Rep. 2005, 22, 15-61; (c) Simmons, T. L.; Andrianasolo, E.; McPhail, K.; Flatt, P.; Gerwick, W. H. Marine natural products as anticancer drugs. Mol. Cancer Ther. 2005, 4, 333-342; (d) Dembitsky, V. M.; Gloriozova, T. A.; Poroikov, V. V. Novel antitumor agents: marine sponge alkaloids, their synthet- 
ic analogs and derivatives. Mini-Rev. Med. Chem. 2005, 5, 319-336; (e) Newman, D. J.; Cragg, G. M. Marine Natural Products and Related Compounds in Clinical and Advanced Preclinical Trials J. Nat. Prod. 2004, 67, 1216-1238; (f) Meth-Cohn, O.; Barton, D.; Nakanishi, K. Compr. Nat. Prod. Chem., Elsevier Science, Oxford, UK, 1999.

(2) For selected examples see: (a) Williamson, A.; Justus, L. Ueber die Theorie der Aetherbildung. Ann. Chem., 1851, 77, 37-49; (b) Krti, L.; Czako, B. Strategic Applications of Named Reactions in Organic Synthesis, Elsevier, Burlington, 2005, pp. 484-485; (c) Alonso, F.; Beletskaya, I. P.; Yus, M. Transition-Metal-Catalyzed Addition of Heteroatom-Hydrogen Bonds to Alkynes. Chem. Rev. 2004, 104, 3079-3160; (d) Bezzenine-Lafollée, S.; Gil, R.; Prim, D.; Hannedouche, J. First-Row Late Transition Metals for Catalytic Alkene Hydrofunctionalisation: Recent Advances in C-N, C-O and C-P Bond Formation. Molecules 2017, 22, 1901; (e) Mann, G.; Hartwig, J. F. Palladium Alkoxides: Potential Intermediacy in Catalytic Amination, Reductive Elimination of Ethers, and Catalytic Etheration. Comments on Alcohol Elimination from $\operatorname{Ir}(\mathrm{III})$. J. Am. Chem. Soc. 1996, 118, 13109-13110; (f) Palucki, M.; Wolfe, J. P.; Buchwald, S. L. Palladium-Catalyzed Intermolecular Carbon-Oxygen Bond Formation: A New Synthesis of Aryl Ethers. J. Am. Chem. Soc. 1997, 119, 3395-3396; (g) Ullmann, F.; Sponagel, P. Ueber die Phenylirung von Phenolen. Chem. Ber. 1905, 38, 2211-2212.

(3) For comprehensive reviews on $\mathrm{sp}^{3} \alpha-\mathrm{C}-\mathrm{H}$ activation of ethers, see: (a) Zhang, S.-Y.; Zhang, F.-M.; Tu, Y.-Q. Direct $\mathrm{Sp}^{3} \alpha-\mathrm{C}-\mathrm{H}$ activation and functionalization of alcohol and ether. Chem. Soc. Rev. 2011, 40, 1937-1949; (b) Guo, S. R.; Pailla, S. K.; Yang, M. H. Recent Advances of Oxidative Radical Cross Coupling Reactions: Direct $\alpha \mathrm{C}\left(\mathrm{sp}^{3}\right)-\mathrm{H}$ Bond Functionalization of Ethers and Alcohols. Adv. Synth. Catal. 2017, 359, 2-25; (c) Qin, Y.; Zhu, L.; Luo, S. Organocatalysis in Inert $\mathrm{C}-\mathrm{H}$ Bond Functionalization, Chem. Rev. 2017, 117, 9433-9520. (d) Liu, C.; Liu, D.; Lei, A. Recent Advances of Transition-Metal Catalyzed Radical Oxidative Cross-Couplings, Acc. Chem. Res. 2014, 47, 3459-3470; (e) Davies, H. M. L.; Beckwith, R. E. J. Catalytic Enantioselective C-H Activation by Means of MetalCarbenoid-Induced C-H Insertion, Chem. Rev. 2003, 103, 2861-2903. (f) Jin, J.; MacMillan, D. W. C. Direct a-Arylation of Ethers through the Combination of Photoredox- Mediated C-H Functionalization and the Minisci Reaction. Angew. Chem. Int. Ed. 2015, 54, 1565-1569; (g) Xia, Q.; Dong, J.; Song, H.; Wang, Q. Visible-Light Photocatalysis of the Ketyl Radical Coupling Reaction. Chem. Eur. J. 2019, 25, 29492961.

(4) (a) Li, C.-J. Exploration of New Chemical Reactivities for Sustainable Molecular Transformations Chem 2016, 1, 423-437; (b) Zhang, C.; Tang, C.; Jiao, N. Recent advances in copper-catalyzed dehydrogenative functionalization via a single electron transfer (SET) process. Chem. Soc. Rev. 2012, 41, 3464-3484; (c) Yeung, C. S.; Dong, V. M. Catalytic Dehydrogenative Cross-Coupling: Forming Carbon-Carbon Bonds by Oxidizing Two Carbon-Hydrogen Bonds Chem. Rev. 2011, 111, 1215-1292; (d) Girard, S. A.; Knauber, T.; Li, C.-J. The Cross-Dehydrogenative Coupling of $\mathrm{Csp}^{3}-\mathrm{H}$ Bonds: A Versatile Strategy for C-C Bond Formations. Angew. Chem. Int. Ed. 2014, 53, 74-100; (e) Wendlandt, A. E.; Stahl, S. S. QuinoneCatalyzed Selective Oxidation of Organic Molecules, Angew. Chem. Int. Ed. 2015, 54, 14638-14658.

(5) For references on the use of DIBAL, see: (a) Takacs, J. M.; Helle, M. A.; Seely, F. L. An improved procedure for the two carbon homologation of esters to $\alpha, \beta$-unsaturated esters. Tetrahedron Lett. 1986, 27, 1257-1260; (b) Burke, S. D.; Deaton, D. N.; Olsen, R. J.; Armistead, D. M.; Blough, B. E. Stereocontrolled one-pot conversions of $\alpha$-alkoxy esters to syn- and anti-1,2-diol derivatives. Tetrahedron Lett. 1987, 28, 3905-3906; (c) Kiyooka, S.; Shirouchi, M. Boron trifluoride promoted aldol reaction of silyl ketene acetals with the intermediate generated by the DIBALH reduction of carboxylic acid esters. J. Org. Chem. 1992, 57, 1-2; (d) Polt, R.; Peterson, M. A.; DeYoung, L. Aluminoxy acetals from .alpha.-amino esters: chirality transfer via sequential addition of hydride and C-nucleophiles. 2Amino alcohols and sphingosines. J. Org. Chem. 1992, 57, 54695480; (e) Kiyooka, S.; Shirouchi, M.; Kaneko, Y. First general synthesis of monosilyl acetals. Trimethylsilyl trapping of the intermediate in DIBALH reduction of carboxylic acid esters using trimethylsilyl trifluoromethanesulfonate. Tetrahedron Lett. 1993, 34, 1491-1494; (f) Ishihara, T.; Hayashi, H.; Yamanaka, H. Zinc bromide promoted allylation of aluminum acetals derived from perfluoro carboxylic acid esters and diisobutylaluminum hydride. New convenient access to $\alpha$ perfluoroalkylated homoallyl alcohols. Tetrahedron Lett. 1993, 34, 5777-5780; (g) Hoye, T. R.; Kopel, L. C.; Ryba, T. D. In situ Generation and Nucleophilic Capture of 1,n-Dial Equivalents from 1,nDioates ( $\alpha, \omega$-Diesters). Synthesis 2006, 10, 1572-1574; (h) Yamazaki, T.; Kobayashi, R.; Kitazume, T.; Kubota, T. In Situ Generation of 3,3,3-Trifluoropropanal and Its Use for Carbon-Carbon BondForming Reactions. J. Org Chem. 2006, 71, 2499-2502.

(6) For further reading on DIBAL see: (a) Boussonnière, A.; Bénéteau, R.; Lebreton, J.; Dénès, F. Aluminum Acetals in Organic Synthesis. Eur. J. Org. Chem. 2013, 7853-7866; (b) Kadota, I.; Yamamoto, Y. Synthetic Strategies of Marine Polycyclic Ethers via Intramolecular Allylations: Linear and Convergent Approaches. Acc. Chem. Res. 2005, 38, 423-432.

(7) For transition metal catalyzed deoxygenative hydrogenation of esters via hydrosilylation see: (a) Berk, S. C.; Kreutzer, K. A.; Buchwald, S. L. A catalytic method for the reduction of esters to alcohols. J. Am. Chem. Soc. 1991, 113, 5093-5095; (b) Berk, S. C.; Buchwald, S. L. An air-stable catalyst system for the conversion of esters to alcohols. J. Org. Chem. 1992, 57, 3751-3753; (c) Barr, K. J.; Berk, S. C.; Buchwald, S. L. Titanocene-Catalyzed Reduction of Esters Using Polymethylhydrosiloxane as the Stoichiometric Reductant. J. Org. Chem. 1994, 59, 4323-4326; (d) Mao, Z.; Gregg, B. T.; Cutler, A. R. Catalytic hydrosilylation of organic esters using manganese carbonyl acetyl complexes. J. Am. Chem. Soc. 1995, 117, 10139-10140; (e) Hansen, M. C.; Verdaguer, X.; Buchwald, S. L. Convenient Two-Step Conversion of Lactones into Cyclic Ethers. J. Org. Chem. 1998, 63, 2360-2361; (f) Ohta, T.; Kamiya, M.; Kusui, K.; Michibata, T.; Nobutomo, M.; Furukawa, I. Rhodium-catalyzed reduction of esters to alcohols using diphenylsilane. Tetrahedron Lett. 1999, 40, 69636966; (g) Igarashi, M.; Mizuno, R.; Fuchikami, T. Ruthenium complex catalyzed hydrosilylation of esters: a facile transformation of esters to alkyl silyl acetals and aldehydes. Tetrahedron Lett. 2001, 42, 2149-2151; (h) Matsubara, K.; Iura, T.; Maki, T.; Nagashima, H. A Triruthenium Carbonyl Cluster Bearing a Bridging Acenaphthylene Ligand: An Efficient Catalyst for Reduction of Esters, Carboxylic Acids, and Amides by Trialkylsilanes. J. Org. Chem. 2002, 67, 49854988; (i) Li, H.; Misal Castro, L. C.; Zheng, J.; Roisnel, T.; Dorcet, V.; Sortais, J.-P.; Darcel, C. Angew. Chem. Int. Ed. 2013, 52, 80458049; (j) Cheng, C.; Brookhart, M. Efficient Reduction of Esters to Aldehydes through Iridium-Catalyzed Hydrosilylation. Angew. Chem. Int. Ed. 2012, 51, 9422-9424; (k) Hua, Y.; Asgari, P.; Avullala, T.; Jeon, J. Catalytic Reductive ortho-C-H Silylation of Phenols with Traceless, Versatile Acetal Directing Groups and Synthetic Applications of Dioxasilines. J. Am. Chem. Soc. 2016, 138, 7982-7991. (1) Bunescu, A.; Butcher, T. W.; Hartwig, J. F. Traceless Silylation of $\beta$ $\mathrm{C}\left(\mathrm{sp}^{3}\right)-\mathrm{H}$ Bonds of Alcohols via Perfluorinated Acetals.. J. Am. Chem. Soc. 2018, 140, 1502-1507; (m) Sree, U.; Bokka, A.; Asgari, P.; Trog, G.; Hua, Y.; Nguyen, H. H.; Rahman, N.; Jeon, J. Lewis Base Activation of Silyl Acetals: Iridium-Catalyzed Reductive Horner-Wadsworth-Emmons Olefination. Org. Lett. 2015, 17, 5792-5795; (n) Corre, Y.; Rysak, V.; Capet, F.; Djukic, J.-P.; AgbossouNiedercorn, F.; Michon, C. Selective Hydrosilylation of Esters to Aldehydes Catalysed by Iridium(III) Metallacycles through Trapping of Transient Silyl Cations. Chem. Eur. J. 2016, 22, 14036-14041; (o) Inamoto, Y.; Kaga, Y.; Nishimoto, Y.; Yasuda, M.; Baba, A. Gallium Trihalide Catalyzed Sequential Addition of Two Different Carbon Nucleophiles to Esters by Using Silyl Cyanide and Ketene Silyl Acetals. Chem. Eur. J. 2014, 20, 11664-11668.

(8) Baba and co-workers report an elegant indium-catalyzed protocol, however selective reactions were only observed using sterically demanding esters, otherwise alcohol products predominated, see: (a) Nishimoto, Y.; Inamoto, Y.; Saito, T.; Yasuda, M.; Baba, A. Triiodide Catalyzed Direct Hydroallylation of Esters. Eur. J. Org. Chem. 2010, 3382-3386. For further application of similar methodology see: (b) Lagoutte, R.; Wilkinson, J. A. A novel one-step method for the reductive allylation of esters and the first total synthesis of $( \pm$ )- 
erythrococcamide B. Tetrahedron Lett. 2010, 51, 6942-6944; (c) Inamoto, Y.; Nishimoto, Y.; Yasuda, M.; Baba, A. Direct Use of Esters in the Mukaiyama Aldol Reaction: A Powerful and Convenient Alternative to Aldehydes. Org. Lett. 2012, 14, 1168-1171. For a C-H activation approach see: (d) Hua, Y.; Asgari, P.; Dakarapu, U. S.; Jeon, J. Reductive arene ortho-silanolization of aromatic esters with hydridosilyl acetals. Chem. Commun. 2015, 51, 3778-3781.

(9) Neither allyl silanes nor allyl boronate esters delivered desired product under these conditions When allyl Grignard reagents were used, competitive allylic alcoholic products predominated.

(10) Mass balance in these reactions is predominately over-reduced ether product with no allylation.

(11) Wang, Z.; Shao, H.; Lacroix, E.; Wu, S.-H.; Jennings, H. J.; Zou, W. Epimerization of 2'-Carbonylalkyl-C-Glycosides via Enolation, $\beta$-Elimination and Intramolecular Cycloaddition. J. Org. Chem. 2003, 68, 8097-8105.

(12) Bondzic, B. P. Eilbracht, P. P., A Novel Access to Tetrahydro$\beta$-Carbolines via One-Pot Hydroformylation/Fischer Indole Synthesis: Rearrangement of 3,3-Spiroindoleninium Cations. Org. Lett. 2008, $10,3433-3436$

(13) Hirano, K.; Yorimitsu, H.; Oshima, K. Nickel-Catalyzed 1,4Addition of Trialkylboranes to $\alpha, \beta$-Unsaturated Esters: Dramatic Enhancement by Addition of Methanol. Org. Lett. 2007, 9, 15411544.

(14) Ney, J. E.; Wolfe, J. P. Palladium Catalyzed Synthesis of N Aryl Pyrrolidines from $\gamma$ ( $N$ Arylamino) Alkenes: Evidence for Chemoselective Alkene Insertion into Pd-N Bonds. Angew. Chem. Int. Ed. 2004, 43, 3605-3608.

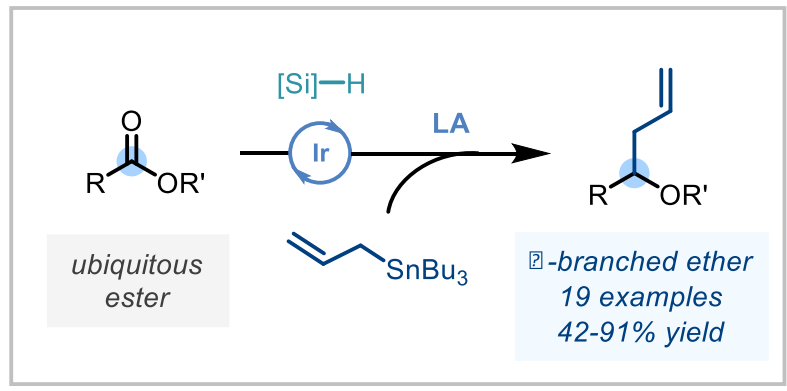

via

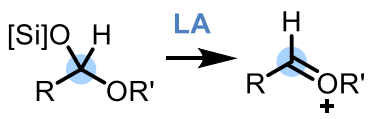

hydrosilylation then oxocarbenium formation 